\title{
Use of Botanicals to Suppress Different Stages of the Life Cycle of Fusarium graminearum
}

\author{
Dimitrios Drakopoulos, ${ }^{1,2}$ Carlos Luz, ${ }^{3}$ Raquel Torrijos, ${ }^{3}$ Giuseppe Meca, ${ }^{3}$ Pascal Weber, ${ }^{1}$ Irene Bänziger, ${ }^{1}$ \\ Ralf T. Voegele, ${ }^{4}$ Johan Six ${ }^{2}$ and Susanne Vogelgsang $1, \dagger$ \\ ${ }^{1}$ Ecological Plant Protection in Arable Crops, Research Division Plant Protection, Agroscope, 8046 Zurich, Switzerland \\ ${ }^{2}$ Sustainable Agroecosystems, Institute of Agricultural Sciences, Department of Environmental Systems Science, ETH Zurich, 8092 \\ ${ }^{3}$ Food Chemistry and Toxicology, Faculty of Pharmacy, University of Valencia, 46100 Burjassot, Spain \\ ${ }^{4}$ Department of Phytopathology, Institute of Phytomedicine, Faculty of Agricultural Sciences, University of Hohenheim, 70599 \\ Accepted for publication 19 July 2019.
} Zurich, Switzerland Stuttgart, Germany

\begin{abstract}
Fusarium head blight (FHB) is one of the most important cereal diseases worldwide, causing yield losses and contamination of harvested products with mycotoxins. Fusarium graminearum is one of the most common FHB-causing species in wheat and barley cropping systems. We assessed the ability of different botanical extracts to suppress essential stages of the fungal life cycle using three strains of $F$. graminearum (FG0410, FG2113, and FG1145). The botanicals included aqueous extracts from white mustard (Sinapis alba) seed flour (Pure Yellow Mustard [PYM] and Tillecur [Ti]) as well as milled Chinese galls (CG). At $2 \%$ concentration (wt/vol), PYM and Ti completely inhibited growth of mycelium of all $F$. graminearum strains whereas, at $1 \%$, CG reduced the growth by 65 to $83 \%$, depending on the strain. While PYM and Ti reduced the germination of both conidia and ascospores at $2 \%(\mathrm{wt} / \mathrm{vol}), \mathrm{CG}$ was only effective in reducing conidia
\end{abstract}

ABSTRACT germination. Perithecia formation of FG0410 but not FG2113 was suppressed by all botanicals. Moreover, application of botanicals on mature perithecia led to a two- to fourfold reduction in discharge of ascospores. Using liquid chromatography (LC) with diode array detection, we quantified the principal glucosinolate component sinalbin of PYM and Ti. LC time-offlight mass spectrometry was used to demonstrate that the bioactive matrix of CG contains different gallotannins as well as gallic and tannic acids. Possible antifungal mechanisms of the botanical matrices are discussed. The results of this study are promising and suggest that PYM, Ti, and CG should be explored further for efficacy at managing FHB.

Keywords: biological control, botanicals, disease control, Fusarium graminearum, mycology
Fusarium head blight (FHB) is a devastating cereal disease worldwide. FHB causes yield losses and can result in severe contaminations of grain with mycotoxins, jeopardizing food and feed safety (McMullen et al. 2012). Worldwide, Fusarium graminearum (teleomorph Gibberella zeae) is one of the most common causes of FHB in wheat and barley cropping systems (Osborne and Stein 2007). F. graminearum produces the mycotoxins deoxynivalenol (DON) and zearalenone (ZEN), which can induce acute and chronic health issues in humans and animals when ingested through contaminated food or feed (Antonissen et al. 2014; Dänicke and Brezina 2013). Chronic effects of DON in animals can result in weight loss, anorexia, and increased susceptibility to facultative pathogens (Marin et al. 2013), while estrogenic effects in swine and cows have been associated with ZEN (D'Mello et al. 1999). Human exposure to DON mainly affects the function of the intestines, immune system, and brain (Escrivá et al. 2015).

†Corresponding author: S. Vogelgsang; susanne.vogelgsang@ agroscope.admin.ch

Funding: This work was supported by the MycoKey project "Integrated and innovative key actions for mycotoxin management in the food and feed chain", which is funded by the Horizon 2020 research and innovation program under the grant agreement number 678781, and the Swiss State Secretariat for Education, Research and Innovation.

*The $\boldsymbol{e}$-Xtra logo stands for "electronic extra" and indicates that two supplementary tables are published online.

The author(s) declare no conflict of interest. distributed under the CC BY-NC-ND 4.0 International license.
The life cycle of $F$. graminearum has been thoroughly studied and described (Dweba et al. 2017; Parry et al. 1995; Trail 2009). Briefly, F. graminearum is an ascomycete with the ability to reproduce both asexually and sexually through conidia and ascospores, respectively. The fungus frequently overwinters on infected crop residues such as maize stalks, mainly as mycelium. In spring, ascospores are forcibly discharged from mature perithecia when weather conditions are favorable (i.e., warm and humid) and infect wheat heads, mostly during anthesis. In contrast, conidia are dispersed on cereal heads and other plant residues by rain-splash. Subsequently, the fungus colonizes the florets and, depending on the severity of infection, kernels develop poorly or not at all. Field management of FHB caused by F. graminearum can be categorized into cultural, chemical, and biological methods (McMullen et al. 2012; Shah et al. 2018). Cultural strategies mainly consist of optimized crop rotations with nonhost crops, tillage practices to bury the crop residues, and use of less susceptible cultivars (Blandino et al. 2012; Gilbert and Haber 2013). Chemical and biological strategies include direct control using fungicides (Amarasinghe et al. 2013) or biocontrol agents (Palazzini et al. 2007, 2017; Zhao et al. 2014), respectively.

Within the context of minimizing the use of conventional crop protection products and to improve food and feed safety, there is an increasing interest in the use of natural, more environmentally friendly plant-based compounds (i.e., botanicals) to control fungal diseases (Dayan et al. 2009; Sellam et al. 2007). Plants belonging to the Brassicaceae family contain glucosinolates, which are secondary metabolites that are hydrolyzed by the enzyme myrosinase into three groups of substances: nitriles, thiocyanates, and isothiocyanates (ITCs) (Zhang and Talalay 1994). The effects of the latter have been investigated extensively and showed antimicrobial, herbicidal, 
antioxidant, and anticancer activity (Hyldgaard et al. 2012; Romeo et al. 2018; Vig et al. 2009). For example, extracts from white mustard (Sinapis alba) seed meal reduced the growth of several fungal species belonging to the genera Fusarium, Aspergillus, and Penicillium, and this effect was attributed to p-hydroxybenzyl ITC (p-HBITC) (Quiles et al. 2018). Chinese galls (CG) (Galla chinensis), a traditional herb rich in gallotannins as well as gallic and tannic acids, are used in human medicine and have also been shown to display antimicrobial activities (Djakpo and Yao 2010; Tian et al. 2009a).

To the best of our knowledge, there have been no studies on the effects of botanicals on different stages of the life cycle of $F$. graminearum. Thus, to better understand the effect of botanicals on critical stages of the life cycle of $F$. graminearum, we tested two mustard-based botanicals and extracts of CG using in vitro bioassays. First, we measured the impacts of the botanicals on mycelium growth, conidia and ascospore germination, development of perithecia on maize stalks, and ascospore discharge from mature perithecia (Fig. 1). Second, we analyzed the chemical composition of the mustard-based botanicals and the CG using liquid chromatography (LC) coupled to diode array detection (DAD) or LC time-offlight mass spectrometry (TOF-MS), respectively.

\section{MATERIALS AND METHODS}

Fungal strains and botanicals. The $F$. graminearum fungal strains FG0410 (CBS121292; Westerdijk Fungal Biodiversity Institute, Utrecht, The Netherlands), FG2113 (Research Group Crop Breeding and Genetic Resources, Plant Pathology, Agroscope, Nyon, Switzerland), and FG1145 (Fungal Collection of Agroscope, Nyon, Switzerland) were isolated from wheat in Switzerland in 2004, 2011, and 1992, respectively. All strains are single-conidial isolates and were identified as 15-acetyldeoxynivalenol genotypes following the methods described by Pasquali et al. (2011) for FG0410 and by Quarta et al. (2006) for FG2113 and FG1145. The botanicals Tillecur (Ti; BIOFA, Münsingen, Germany), Pure Yellow Mustard (PYM; product 106; G.S. Dunn, Dry Mustard Millers, Hamilton, ON, Canada), and CG (origin Sichuan, PR China; purchased from BergApotheke, Zurich, Switzerland) were used in this study and were purchased in a powder form. In organic agriculture, Ti is applied as seed treatment in cereals to protect against common bunt (Tilletia caries, synonym $T$. tritici). Ti and PYM are based on white mustard seed flour. Among other uses, CG is a raw material for industrial production of tannic acid, and a detailed description of this botanical is provided by Tian et al. (2009b). Preliminary experiments were performed in order to determine the final protocols and the range in botanical concentrations for the bioassays.

Mycelium growth and pH. Ti and PYM were tested at $0.5,1$, and $2 \%(\mathrm{wt} / \mathrm{vol})$ and $\mathrm{CG}$ at 0.5 and $1 \%(\mathrm{wt} / \mathrm{vol})$. Potato dextrose agar (PDA; Oxoid Ltd., Basingstoke, U.K.) was prepared in Schott flasks and autoclaved at $121^{\circ} \mathrm{C}$ for $15 \mathrm{~min}$. Flasks were cooled to $50^{\circ} \mathrm{C}$ before adding streptomycin sulfate (Sigma-Aldrich, St. Louis, MO, U.S.A.) at $0.1 \mathrm{~g} \mathrm{liter}^{-1}$ and the desired quantities of botanicals (as powder). Subsequently, $20 \mathrm{ml}$ of each PDA-botanical mixture was poured into Petri dishes (94 by $16 \mathrm{~mm}$; Greiner Bio-One, Kremsmünster, Austria). There were four replicates of each treatment and a control on PDA without botanicals. Mycelial plugs of $0.5 \mathrm{~cm}$ in diameter were cut using a cork borer from the margins of 7- to 14-day-old F. graminearum colonies, grown at $18^{\circ} \mathrm{C}$ with a photoperiod of $12 \mathrm{~h}$ under near-UV light, and placed in the center of each dish with the mycelial side facing the agar. Dishes were incubated in the dark at $20^{\circ} \mathrm{C}$ and $80 \%$ relative humidity (RH). After 6 to 8 days, when the fastest growing fungal colony had covered $90 \%$ of the surface, the colony diameter was measured with a ruler. The bioassay was conducted twice for all strains. The $\mathrm{pH}$ of the botanicals was measured after suspending in deionized water $\left(\mathrm{dH}_{2} \mathrm{O}\right)$ and after incorporation in PDA with a $\mathrm{pH}$-meter (Hanna Instruments Inc., Smithfield, RI, U.S.A.).

Conidia germination. Fresh cultures of FG0410, FG2113, and FG1 145 were produced at $18^{\circ} \mathrm{C}$ with a 12 -h photoperiod under nearUV light. Conidial suspensions of each strain were prepared by flushing 7- to 10-day-old cultures with $\mathrm{dH}_{2} \mathrm{O}$. The surface of the colonies was agitated using a Drigalski spatula and conidial suspensions were adjusted to $2 \times 10^{5}$ conidia $\mathrm{ml}^{-1}$ using a hemocytometer (Thoma Counting Chamber; Paul Marienfeld $\mathrm{GmbH} \&$ Co. KG, Lauda-Königshofen, Germany). Botanical treatments were tested at $0.5,1$, and $2 \%$ (wt/vol). Botanical powders were suspended in sterile $\mathrm{dH}_{2} \mathrm{O}$ and stirred with a magnet for $2 \mathrm{~h}$ at room temperature. A microscope slide ( 76 by 26 by $1 \mathrm{~mm}$; Paul Marienfeld GmbH \& Co. KG) was placed in a Petri dish on a moistened filter paper (8.5 cm in diameter, Nr. 591; Schleicher \& Schuell BioScience GmbH, Dassel, Germany). Four agar plugs, each $1 \mathrm{~cm}$ in diameter $(20 \mathrm{~g}$ of agar agar pulver [ERNE Surface $\mathrm{SA}$, Avenches, Switzerland], amended with streptomycin sulfate [Sigma-Aldrich] at $0.1 \mathrm{~g} /$ liter of $\mathrm{dH}_{2} \mathrm{O}$ ), were placed on the microscope slide and served as replicates. A $15-\mu \mathrm{l}$ aliquot of each botanical suspension was pipetted onto each plug. For the control treatment, sterile $\mathrm{dH}_{2} \mathrm{O}$ was used. The surfaces of the agar plugs were allowed to dry and a $15-\mu \mathrm{l}$ aliquot of the conidial suspension was pipetted onto each plug. Closed dishes were incubated in the dark at $14^{\circ} \mathrm{C}$ and $70 \% \mathrm{RH}$ for $18 \mathrm{~h}$. Conidia were killed by applying $15 \mu \mathrm{l}$ of fungicide $(0.19 \%$ Pronto Plus; Bayer Schweiz AG, Zollikofen, Switzerland) per agar plug. Germination rates were assessed with the aid of a light microscope ( $\times 400$ magnification) by determining the ratio of germinated and nongerminated conidia in a sample of 40 conidia/plug in four to six fields of vision. A conidium was defined as germinated when the germination tube was longer than its width. The bioassay was conducted twice for all strains.

In vitro bioassays performed in this study

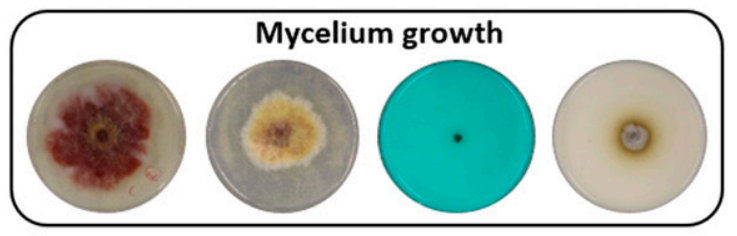

Perithecia formation on maize stalks

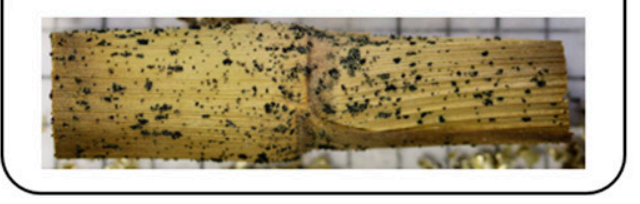

Fig. 1. Components of the life cycle of Fusarium graminearum targeted to assess the suppressive effect of different botanicals using in vitro bioassays.

\section{Ascospore discharge}

Ascospore
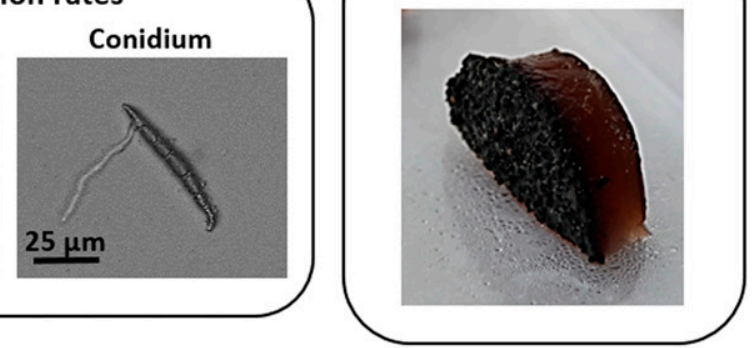

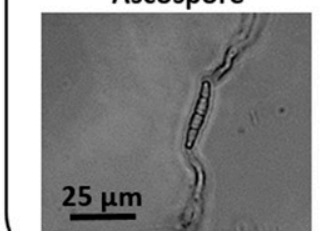


Ascospore germination. Production of perithecia from strains FG0410 and FG2113 was performed following a modified protocol (Klittich and Leslie 1988; Manstretta et al. 2016) using carrot agar as the culture medium. The strain FG1145 was not included due to its very low ascospore germination rate. Fresh organic carrot roots (400 g) were washed, diced, and autoclaved for $15 \mathrm{~min}$ at $121^{\circ} \mathrm{C}$ in $400 \mathrm{ml}$ of $\mathrm{dH}_{2} \mathrm{O}$. Autoclaved carrot roots were pureed, mixed with an additional $500 \mathrm{ml}$ of $\mathrm{dH}_{2} \mathrm{O}$ and $20 \mathrm{~g}$ of agar, and autoclaved again. After cooling to $50^{\circ} \mathrm{C}$, streptomycin sulfate (Sigma-Aldrich) at $0.1 \mathrm{~g}$ liter $^{-1}$ was added. Aliquots of $12 \mathrm{ml}$ of the medium were dispensed into small Petri dishes ( 60 by $15 \mathrm{~mm}$ ). Petri dishes were inoculated with the desired strain of $F$. graminearum by placing a $0.5-\mathrm{cm}-$ diameter agar plug in the center of each dish. Agar plugs were obtained from 5- to 7-day-old cultures of $F$. graminearum growing on PDA incubated at $18^{\circ} \mathrm{C}$ with a 12 -h photoperiod under near-UV light. After inoculation, Petri dishes were incubated in the dark at $18^{\circ} \mathrm{C}$ for 4 to 6 days until mycelium had reached the outer edge of the dish. Subsequently, the aerial mycelium was gently removed under sterile conditions and $1 \mathrm{ml}$ of $2.5 \%$ Tween20 (Sigma-Aldrich) was uniformly spread on the agar surface with a Drigalski spatula. The agar surface was allowed to dry before closing the dishes, which were incubated at $18^{\circ} \mathrm{C}$ with a 12 -h photoperiod under near-UV light. After 1 to 2 weeks, ascospore discharge began from mature perithecia and ascospores were dispersed onto the inner lid surface of the Petri dishes (Schöneberg et al. 2015). Ascospores were collected by washing the inner surface of each lid twice with $1 \mathrm{ml}$ of sterile $\mathrm{dH}_{2} \mathrm{O}$, and adjusting the suspension to $3 \times 10^{5}$ ascospores $\mathrm{ml}^{-1}$. Botanicals were tested at $0.5,1$, and $2 \%$ (wt/vol) and the ascospore germination assessment was performed following the same protocol as for conidia. The bioassay was conducted twice for each strain.

Perithecia formation on maize stalks. Maize stalks were collected from a field at the Agroscope-Reckenholz research station in Zurich, Switzerland. Stalks were dried at $30^{\circ} \mathrm{C}$ for 5 days and cut into $5-\mathrm{cm}$ pieces, which included one node in the midpoint. Each stalk piece was cut in half longitudinally. Subsequently, stalks were immersed overnight in $\mathrm{dH}_{2} \mathrm{O}$, autoclaved twice for $15 \mathrm{~min}$ at $121^{\circ} \mathrm{C}$ and cooled to room temperature. The bioassay was conducted with strains FG0410 and FG2113 (FG1145 was not included due to its inability to produce sufficient perithecia). Autoclaved stalks were inoculated with a suspension containing $2 \times 10^{5}$ conidia ml-1 and $0.0125 \%$ Tween 20 , which was stirred for $5 \mathrm{~min}$. Botanical powders were dissolved in $500 \mathrm{ml}$ of sterile $\mathrm{dH}_{2} \mathrm{O}(2 \%$ [wt/vol] $)$ and stirred for $2 \mathrm{~h}$. After inoculation, stalks were immersed in the solution containing the botanicals and stirred for $2 \mathrm{~min}$. For the water control, stalks were immersed in sterile $\mathrm{dH}_{2} \mathrm{O}$ and stirred for 2 min. For the control, stalks were not immersed in either water or botanical solution. Following the treatment application, two stalks were placed into a Petri dish (140 by $20 \mathrm{~mm})$ containing $20 \mathrm{~g}$ of water-saturated autoclaved vermiculite (ISOLA Vermiculite AG, Bözen, Switzerland) and incubated at $18^{\circ} \mathrm{C}$ with a 12 -h photoperiod under near-UV light for approximately 3 weeks, until perithecia were formed. One Petri dish represented one replicate and each treatment included six replicates. Images of stalk sections were captured with a digital camera (Canon EOS 750D) using a 60-mm EFS macro lens. Perithecia were counted along the full length of the stalk following a 1-cm-wide transect centered on the middle of the longitudinally cut half stalk. Average values of perithecia on both stalk sections per Petri dish were calculated for each replicate. The bioassay was conducted twice for each strain.

Ascospore discharge. The ascospore discharge bioassay protocol of Trail et al. (2002) was modified to suit the requirements of the $F$. graminearum strains that were used. The bioassay was conducted with strains FG0410 and FG2113. Perithecia were produced on carrot agar using the protocol described in the previous section. When perithecia were mature, carrot agar plugs $(1 \mathrm{~cm}$ in diameter) were removed with a cork borer and the agar cores were split longitudinally using a surgical blade ("half-plugs"). Four half-plugs were placed flat side down on the surface of a microscope slide ( 76 by 26 by $1 \mathrm{~mm}$ ).
Botanical powders were suspended in sterile $\mathrm{dH}_{2} \mathrm{O}(2 \%$ [wt/vol] $)$ and stirred for $2 \mathrm{~h}$ at room temperature. Aliquots of $20 \mu \mathrm{l}$ of botanical suspension or sterile $\mathrm{dH}_{2} \mathrm{O}$ for the control were applied directly on the upper semicircle side of each half-plug. The microscope slides were placed on moistened filter paper in transparent plastic boxes ( 80 by 50 by $25 \mathrm{~mm}$ ). One box with four half-plugs represented one replicate, with six replicates of each treatment. Closed boxes were incubated at $20^{\circ} \mathrm{C}$ for $24 \mathrm{~h}$ under continuous near-UV light. Halfplugs were removed and the discharged ascospores were collected by washing each slide with $1 \mathrm{ml}$ of sterile $\mathrm{dH}_{2} \mathrm{O}$ using a pipette, and transferring the solution into 2-ml Eppendorf tubes. Ascospores were quantified with a hemocytometer. The bioassay was conducted twice.

Glucosinolate extraction and determination in mustards. Glucosinolates from PYM and $\mathrm{Ti}$ were extracted using a modified method from Prestera et al. (1996). Both matrices were subjected to an aqueous extraction and analyzed using LC-DAD. Botanical powders ( $20 \mathrm{~g}$ each) were placed into a $50-\mathrm{ml}$ glass tube and autoclaved at $115^{\circ} \mathrm{C}$ for $15 \mathrm{~min}$ to inactivate the enzyme myrosinase. Samples, together with $200 \mathrm{ml}$ of boiling distilled water, were added to a 500-ml Erlenmeyer flask and the mixture was stirred for $10 \mathrm{~min}$ at $350 \mathrm{rpm}$. Mixtures were allowed to cool down to room temperature, centrifuged at 2,500 rpm for $5 \mathrm{~min}$ at $4{ }^{\circ} \mathrm{C}$, and filtered through Whatman number 4 filter paper into $50-\mathrm{ml}$ screw-capped tubes. The extracts were filtered again through a $0.22-\mu \mathrm{m}$ filter. Separation and quantification of glucosinolates were performed using a Shimadzu LC system (Shimadzu, Kyoto, Japan), equipped with a Gemini C18 column ( 4.6 by $150 \mathrm{~mm}$, internal diameter $=5 \mathrm{~mm}$; Phenomenex, Torrance, CA, U.S.A.). Elution was carried out isocratically for $20 \mathrm{~min}$ at a flow rate of $1 \mathrm{ml} \mathrm{min}^{-1}$, using $20 \%$ (vol/vol) acetonitrile and $80 \%$ water with $0.02 \mathrm{M}$ tetrabutylammonium hydrogen sulfate $(\mathrm{pH} 5.5)$. The injection volume was set to $20 \mu \mathrm{l}$. A UV detector was used to measure the absorbance at $227 \mathrm{~nm}$ in order to verify and quantify the presence of sinalbin at a reference retention time of $1.83 \mathrm{~min}$.

Separation and identification of gallotannins in CG. The CG powder was suspended in water at $4 \%(\mathrm{wt} / \mathrm{vol})$ and vortexed for $1 \mathrm{~min}$. The mixture was centrifuged for $10 \mathrm{~min}(3,000 \mathrm{rpm})$ and the supernatant was transferred to a $15-\mathrm{ml}$ plastic tube. The extract was filtered with a $0.22-\mu \mathrm{m}$ Whatman filter into an LC amber vial. An Agilent 1200-LC system (Agilent Technologies, Palo Alto, CA, U.S.A.) equipped with vacuum degasser, autosampler, and binary pump was used for the chromatographic determination. The column was a Gemini NX-C18 (2 mm ^ by approximately $110 \mathrm{~mm}$, particle size $=3 \mu \mathrm{m}$; Phenomenex). Mobile phases consisting of $0.1 \%$ formic acid as solvent system A and acetonitrile as solvent system $\mathrm{B}$ were used with the following gradient elution: $0 \mathrm{~min}, 5 \% \mathrm{~B} ; 30 \mathrm{~min}, 95 \% \mathrm{~B} ; 35 \mathrm{~min}, 95 \% \mathrm{~B}$; and $40 \mathrm{~min}, 5 \% \mathrm{~B}$. The column was equilibrated for $3 \mathrm{~min}$ prior to each analysis. The sample volume injection was $20 \mu \mathrm{l}$ and the flow rate was $0.3 \mathrm{ml} \mathrm{min}^{-1}$. MS analysis was carried out using a 6540 Agilent Ultra-High-Definition Accurate-Mass q-TOF-MS coupled to the high-performance liquid chromatography, equipped with an Agilent Dual Jet Stream electrospray ionization (Dual AJS ESI) interface in negative ionization mode. The conditions were as follows: drying gas flow $\left(\mathrm{N}_{2}\right), 12$ liter $\mathrm{min}^{-1}$; nebulizer pressure, 50 psi; gas drying temperature, $350^{\circ} \mathrm{C}$; capillary voltage, $3,500 \mathrm{~V}$; and fragmentor voltage, and scan range were $200 \mathrm{~V}$ and $\mathrm{m} / \mathrm{z} 50$ to 3,000, respectively. Automatic tandem MS experiments were carried out using collision energy values of 0,20 , and $40 \mathrm{eV}$. Integration and data elaboration were performed using the Mass Hunter Workstation software (Agilent Technologies).

Statistical analysis. Data were analyzed with the statistical software SigmaPlot 13.0 (Systat Software Inc., San Jose, CA, U.S.A.) and figures were prepared with Prism 7.0 (GraphPad Software Inc., San Diego, CA, U.S.A.). To check for normality and homogeneity of variances, data were subjected to Shapiro-Wilk and Brown-Forsythe tests, respectively. When both tests passed $(P>$ 0.05 ), a parametric one-way analysis of variance (ANOVA) was 
conducted. Otherwise, the nonparametric Kruskal-Wallis one-way ANOVA on ranks was performed. All pairwise comparisons among treatments were conducted using the Student-Newman-Keuls method with an $\alpha$ value of 0.05 . For each study, the effect of the interaction experimental run $\times$ treatment was tested before pooling the data from the two runs of each bioassay. The interactions were not significant for most variables.

\section{RESULTS}

Mycelium growth and pH. PYM fully inhibited mycelium growth of FG0410 and FG2113 at $1 \%$ (wt/vol $)(P<0.001)$, while Ti resulted in a full inhibition at $2 \%(\mathrm{wt} / \mathrm{vol})(P<0.001)$ (Fig. 2$)$.
Compared with the control, $\mathrm{CG}$ at $1 \%$ (wt/vol) suppressed mycelium growth of FG0410 by $82 \%(P=0.009)$ and FG2113 by $83 \%(P<$ 0.001) (Fig. 2). At $0.5 \%$ (wt/vol), Ti and PYM increased mycelium growth of FG1145 $(P<0.001$ and $P=0.005$, respectively). However, full inhibition was observed at $2 \%$ (wt/vol) $(P<0.001)$ (Fig. 2). CG at $1 \%(\mathrm{wt} / \mathrm{vol})$ reduced mycelium growth of FG1145 by $65 \%$ compared with the control $(P<0.001)$ (Fig. 2). The pH of PDA in $\mathrm{dH}_{2} \mathrm{O}$ was 5.6. The $\mathrm{pH}$ of Ti and $\mathrm{PYM}$ suspensions in $\mathrm{dH}_{2} \mathrm{O}$ and after PDA incorporation ranged from 5.0 to 5.5 whereas, with $\mathrm{CG}$, lower $\mathrm{pH}$ values (3.9 to 4.5) were observed (Supplementary Table S1).

Conidia germination. At $2 \%$ (wt/vol), all botanicals inhibited germination of most conidia of all three strains of $F$. graminearum $(P<0.001)$ (Fig. 3). At $0.5 \%$ (wt/vol), CG substantially decreased
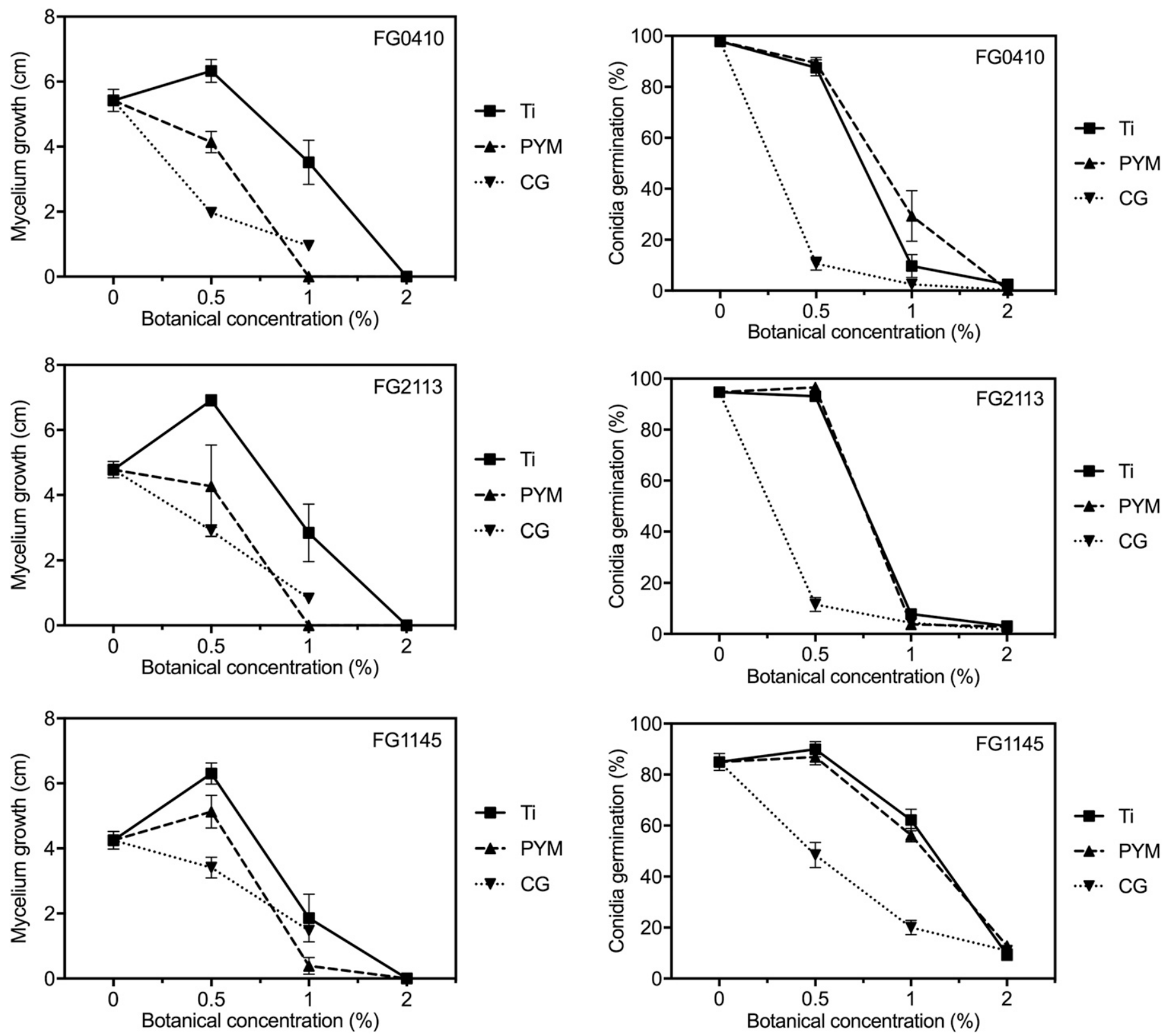

Fig. 2. Mycelium growth (in centimeters) of three strains of Fusarium graminearum (FG0410, FG2113, and FG1145) as affected by different concentrations (wt/vol) of the botanicals Tillecur (Ti), Pure Yellow Mustard (PYM), and Chinese galls (CG). The control is indicated as $0 \%$ botanical concentration. Average values from two experimental runs are presented and bars indicate the standard error of the mean $(n=8)$. Results from a KruskalWallis test were significant for FG0410 $\left(\chi^{2}=66.0\right.$, $\left.\mathrm{df}=8, P<0.001\right)$, FG2113 $\left(\chi^{2}=53.2, \mathrm{df}=8, P<0.001\right)$, and FG1145 $\left(\chi^{2}=59.9, \mathrm{df}=8, P<\right.$ $0.001)$. All pairwise comparisons were performed using a Student-NewmanKeuls test $(\alpha=0.05)$.

Fig. 3. Conidia germination (percent) of three strains of Fusarium graminearum (FG0410, FG2113, and FG1145) as affected by different concentrations (wt/vol) of the botanicals Tillecur (Ti), Pure Yellow Mustard (PYM), and Chinese galls (CG). The control is indicated as $0 \%$ botanical concentration. Average values from two experimental runs are presented and bars indicate the standard error of the mean $(n=8)$. Results from a Kruskal-Wallis test were significant for FG0410 $\left(\chi^{2}=64.0, \mathrm{df}=9, P<0.001\right)$. Results from a one-way analysis of variance were significant for FG2113 $\left(\mathrm{F}_{9,70}=781.3, P<0.001\right)$ and FG1145 $\left(\mathrm{F}_{9,70}=112.4, P<0.001\right)$. All pairwise comparisons were performed using a Student-Newman-Keuls test $(\alpha=0.05)$. 
conidia germination (FG0410 by $89 \%$, FG2113 by $88 \%$, and FG1145 by $43 \%)$ compared with the control $(P<0.001)$, while Ti and PYM were more effective at $\geq 1 \%$ (wt/vol) $(P<0.001)$ (Fig. 3$)$.

Ascospore germination. Ti and PYM at $\geq 1 \%$ (wt/vol) inhibited ascospore germination of FG0410 and FG2113 ( $P<0.001)$ (Fig. 4). At all concentrations tested, $C G$ decreased ascospore germination of FG0410 only slightly compared with the control (4 to $8 \% ; P<0.01$ ), and no effect was observed with FG2113 (Fig. 4).

All pairwise comparisons among treatments with the corresponding $P$ values for the bioassays of mycelium growth, conidia germination, and ascospore germination are provided in Supplementary Table S2.

Perithecia formation on maize stalks. Ti, PYM, and CG at $2 \%(\mathrm{wt} / \mathrm{vol})$ suppressed perithecia formation of FG0410 on maize stalks by 48,56 , and $53 \%$, respectively, compared with the water control $(P<0.001)$, whereas the effects on FG2113 were not significant $(P>0.05)$ (Fig. 5). For both FG0410 and FG2113, there was no difference in the number of perithecia on maize stalks between the control and water control $(P>0.05)$ (Fig. 5).

Ascospore discharge. The number of ascospores discharged from mature perithecia produced on carrot agar was reduced with application of Ti, PYM, and CG at $2 \%$ (wt/vol) (Fig. 6). With FG0410, 77, 59, and 58\% fewer ascospores $(P<0.01)$ and, with FG2113, 70, 54, and 62\% fewer ascospores $(P<0.001)$ were discharged when mature perithecia were treated with Ti, PYM, or CG, respectively (Fig. 6).

Glucosinolate determination in mustards and gallotannin identification in CG. Glucosinolates present in PYM and Ti were analyzed to determine the total quantity that could be converted into ITCs through the action of the enzyme myrosinase.
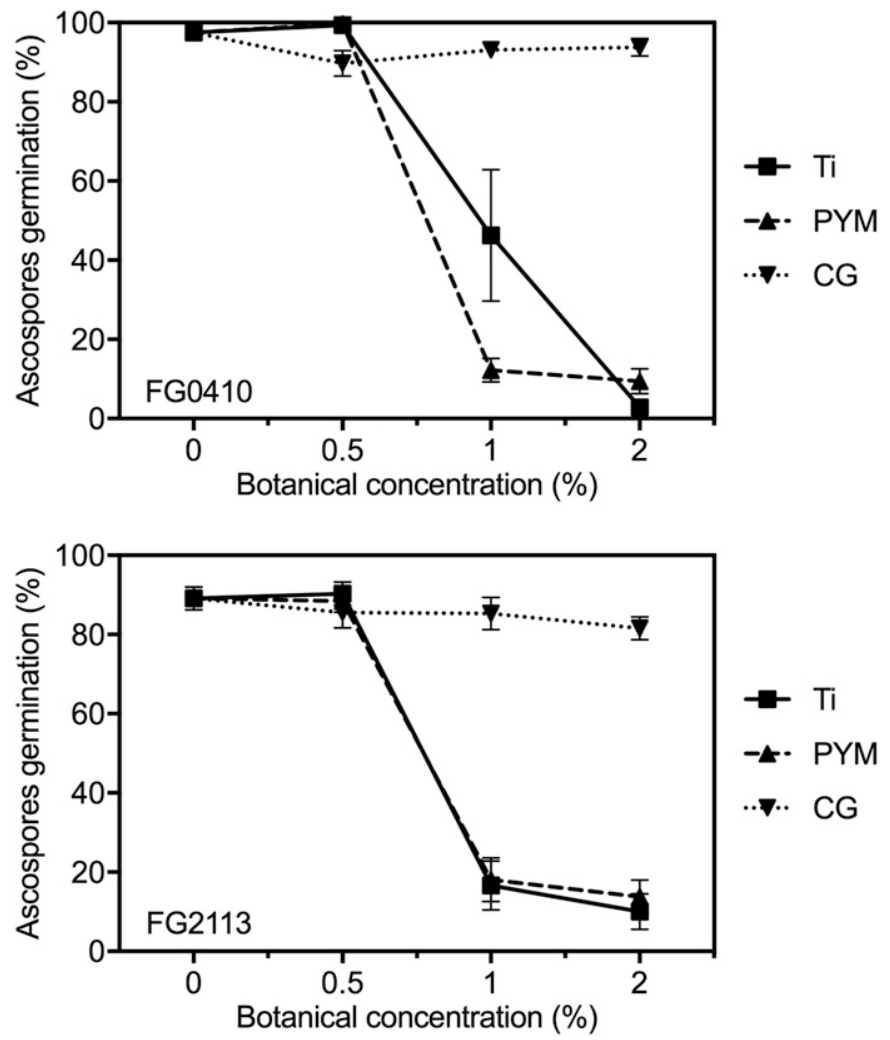

Fig. 4. Ascospore germination (percent) of two strains of Fusarium graminearum (FG0410 and FG2113) as affected by different concentrations (wt/vol) of the botanicals Tillecur (Ti), Pure Yellow Mustard (PYM), and Chinese galls (CG). The control is indicated as $0 \%$ botanical concentration. Average values from two experimental runs are presented and bars indicate the standard error of the mean $(n=8)$. Results from a Kruskal-Wallis test were significant for FG0410 $\left(\chi^{2}\right.$ $=62.7, \mathrm{df}=9, P<0.001)$ and FG2113 $\left(\chi^{2}=59.1, \mathrm{df}=9, P<0.001\right)$. All pairwise comparisons were performed using a Student-Newman-Keuls test $(\alpha=0.05)$.
The principal glucosinolate present in PYM and Ti was sinalbin, the powders containing 57.4 and $56.4 \mathrm{~g} \mathrm{~kg}^{-1}$, respectively. Analysis of gallotannins present in CG(CGTs), using LC-TOF-MS, demonstrated that this bioactive matrix contained 14 different compounds; that were, tannic acid (54\%), gallic acid (19\%), and 12 different gallotannins (Table 1). Most of the CGTs had fragmentation profiles from the loss of one or more galloyl groups (152 mass units) or the loss of gallic acid (170 mass units). Some isomers of 4-galloylglucopyranose (GG) and 5-GG gave the fragmentation of digallic acid $(\mathrm{m} / \mathrm{z}=$ 321 ), which indicates that not all of the galloyl groups of these isomers are directly attached to the glucose core. Some of them might be attached to another galloyl group via a meta-depside bond. The molecular weight (MW) of the identified compounds ranged from low-MW compounds such as gallic acid, with an MW of 169.0560, to organic compounds with an MW ranging from 635.1750 to 1,243.2302 (molecules identified as 3-, 4-, 5-, 6-, and 7-GG). In addition, other compounds with high MW (i.e., 12-, 14-, and 15-GG) ranging from $2,004.3014$ to $2,460.3391$ were identified.

\section{DISCUSSION}

We believe this is the first report describing the use of botanicals to suppress $F$. graminearum by assessing the impact on multiple, distinct stages of the pathogen life cycle. The botanicals used for
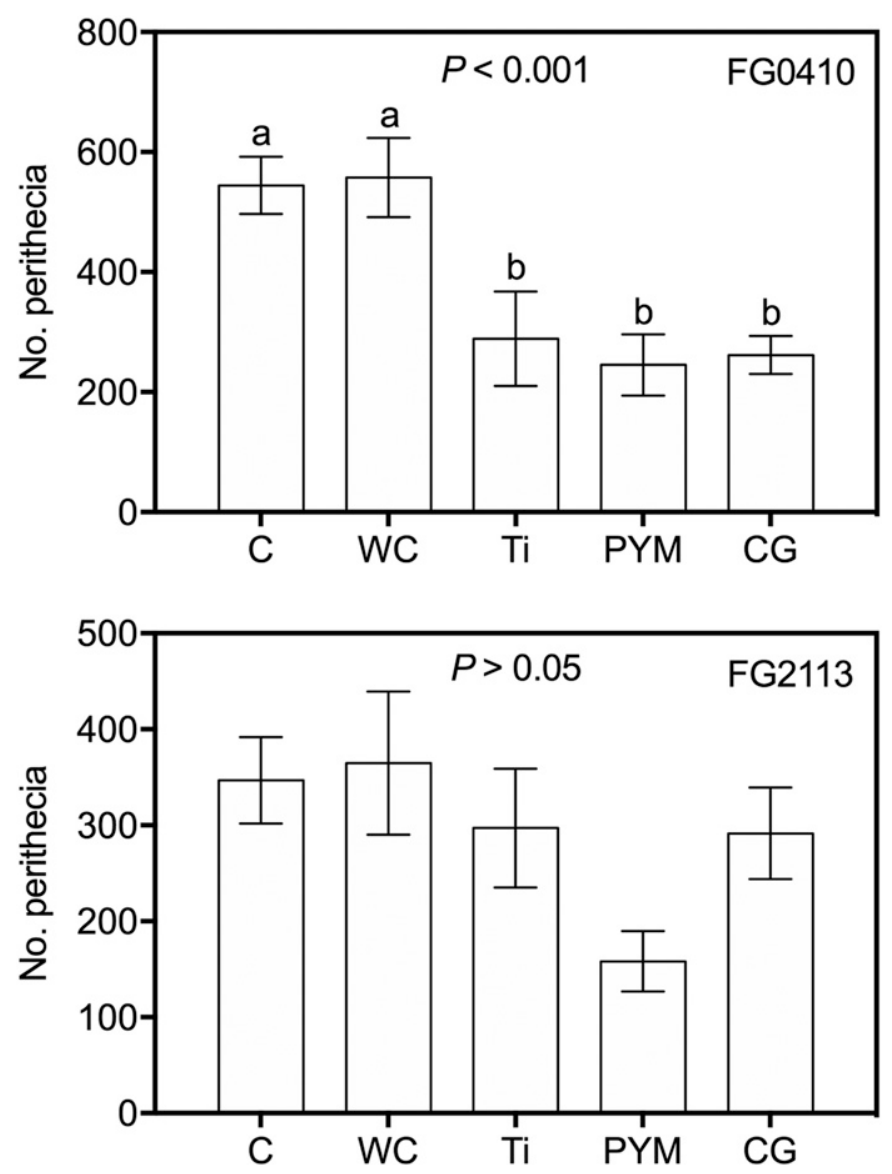

Fig. 5. Number of perithecia formed on maize stalks by two strains of Fusarium graminearum (FG0410 and FG2113) as affected by Tillecur (Ti), Pure Yellow Mustard (PYM), and Chinese galls (CG) at 2\% concentration (wt/ vol). The control (C) and water control (WC) refer to untreated stalks and stalks treated with sterile deionized water, respectively. Average values from two experimental runs are presented and bars indicate the standard error of the mean $(n=12)$. Results from a Kruskal-Wallis test were significant for FG0410 $\left(\chi^{2}=22.7, \mathrm{df}=4, P<0.001\right)$. All pairwise comparisons were performed using a Student-Newman-Keuls test and different letters indicate significant differences $(\alpha=0.05)$. Results from a one-way analysis of variance were not significant for FG2113 $\left(\mathrm{F}_{4,55}=2.2, P>0.05\right)$. 

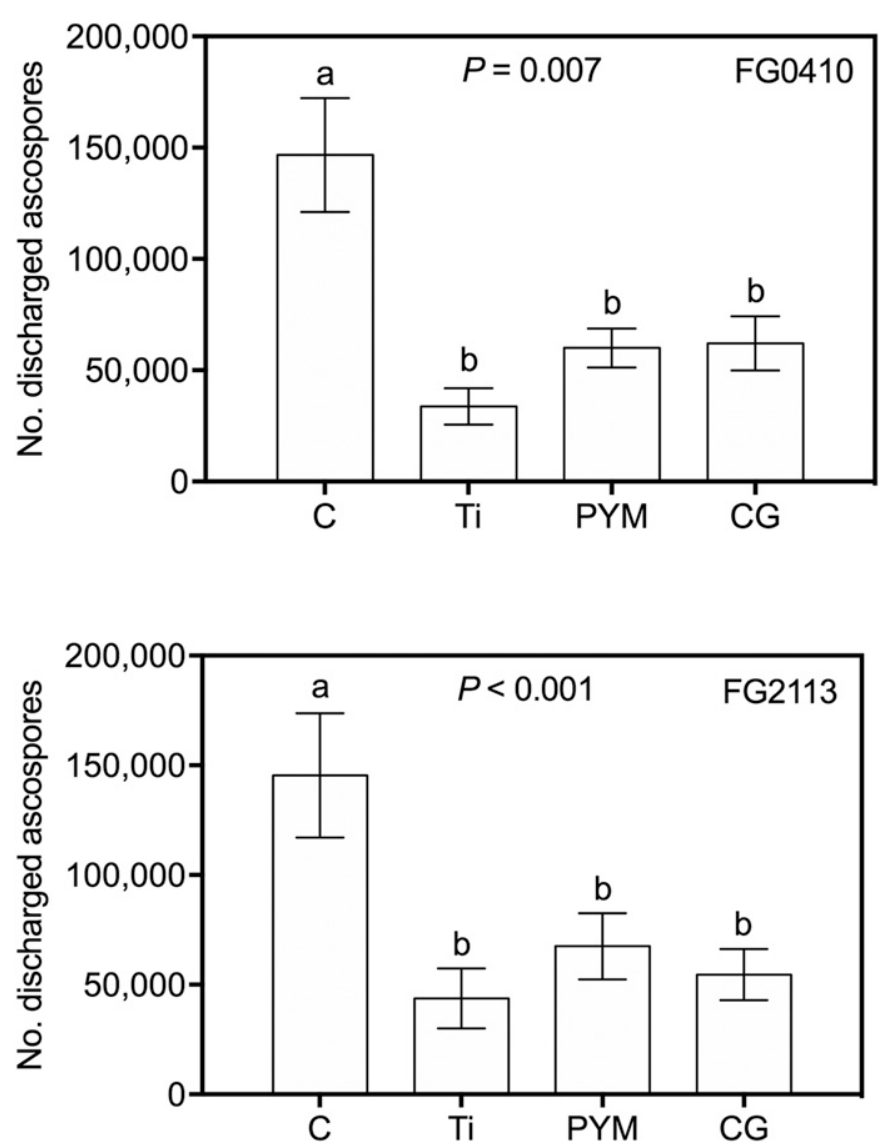

Fig. 6. Number of ascospores discharged by two strains of Fusarium graminearum (FG0410 and FG2113) as affected by Tillecur (Ti), Pure Yellow Mustard (PYM), and Chinese galls (CG) at $2 \%$ concentration (wt/vol). For the control (C), sterile deionized water was applied. Average values from two experimental runs are presented and bars indicate the standard error of the mean $(n=12)$. Results from a Kruskal-Wallis test were significant for FG0410 $\left(\chi^{2}=12.3, \mathrm{df}=3, P<0.01\right)$ and FG2113 $\left(\chi^{2}=15.7, \mathrm{df}=3, P<0.001\right)$. All pairwise comparisons were performed using a Student-Newman-Keuls test and different letters indicate significant differences $(\alpha=0.05)$. this research included PYM and Ti, which are both based on white mustard seed flour, and CG, which is rich in gallotannins as well as in gallic and tannic acid. All botanicals tested showed promise, suppressing the development of structures at different life-cycle stages of three strains of $F$. graminearum. The only exception was CG, which showed only minor or no effects on suppressing ascospore germination, depending on the fungal strain. Overall, the bioassays on mycelium growth, conidia and ascospore germination, and ascospore discharge showed similar patterns among all $F$. graminearum strains examined. However, a differential response was observed in reduction of the number of perithecia on maize stalks; treatment with botanicals reduced the number of perithecia for FG0410 but not for FG2113. The difference in response may indicate that prevention of perithecial development may be inconsistent when applying these botanicals on maize residues but, clearly, further research is needed to ascertain these effects at the field scale.

The forcible discharge of ascospores from mature perithecia represents the initial inoculum and, therefore, is a key element for FHB infection. Hence, interruption of ascospore discharge in $F$. graminearum might be an effective method to control FHB, as has been previously reported for ascomycetes that rely on ascospores for primary inoculum (Trail 2007). In the current study, we observed that ascospore discharge from mature perithecia was two- to fourfold lower after application of botanicals. After deposition on flowering cereal heads, the germination of ascospores and conidia are the major developmental steps in the pathogenesis of FHB (Dweba et al. 2017). We showed that the germination of both spore types was completely inhibited in vitro by applying PYM or Ti. Although application of CG suppressed conidial germination, it did not have a sufficiently suppressive effect against ascospore germination, indicating that reduction in activity of these spores may be more difficult to achieve. Another critical stage is the survival of $F$. graminearum, which overwinters as mycelium in soil and on infected crop residues (Leplat et al. 2013; Yuen and Schoneweis 2007). Therefore, reducing the mass of hyphae left in the field could be an effective strategy to further minimize the risk of FHB epidemics. All botanicals tested could be candidates for inclusion in a management strategy to reduce overwintering mycelium in the soil and on infected crop residues. All three botanicals consistently suppressed the mycelium growth of all three strains of $F$. graminearum at concentrations $\geq 1 \%$.

TABLE 1. Negative ion liquid chromatography electrospray ionization quantitative time-of-flight mass spectrometry (LC-ESI-qTOF-MS) analysis of gallotannins in extracts of Chinese galls

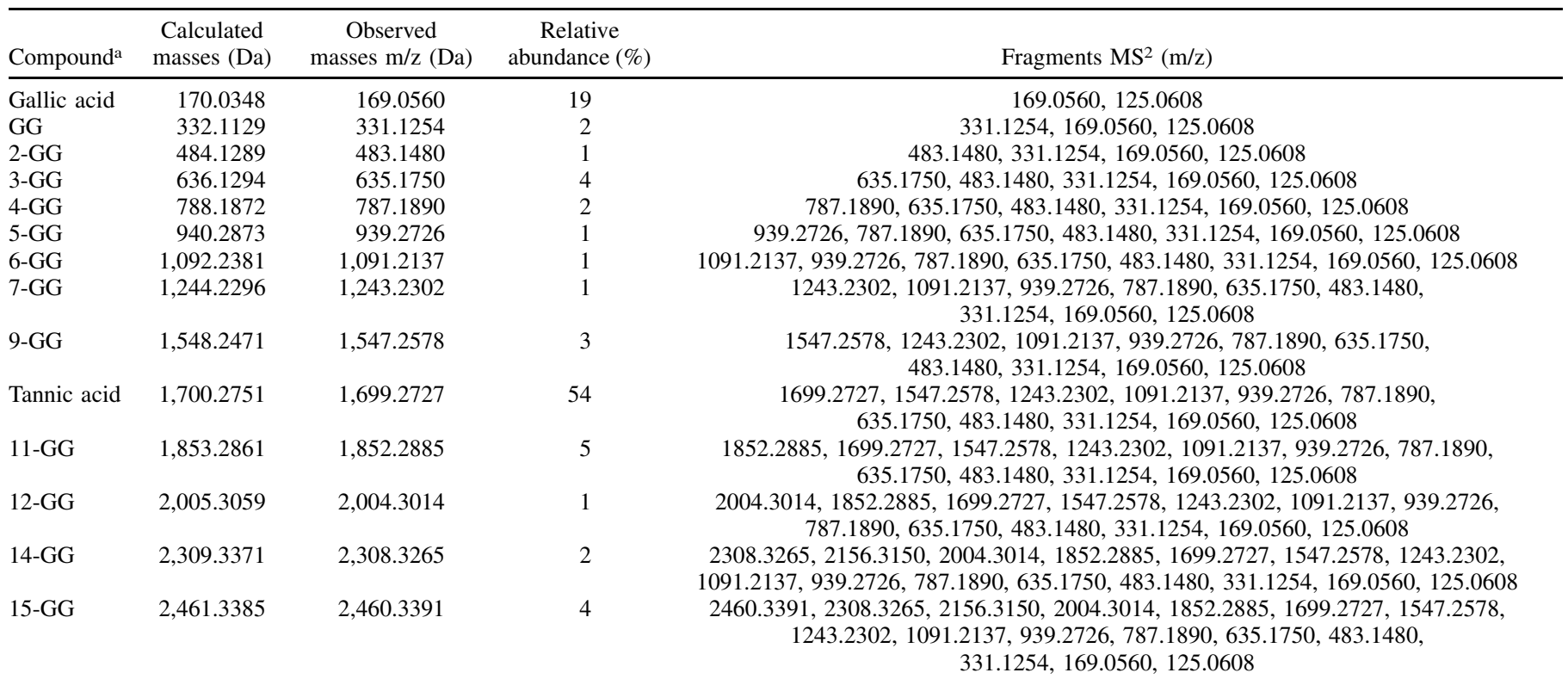

${ }^{\mathrm{a}} \mathrm{GG}$ = galloylglucopyranose. 
Sinalbin was the principal glucosinolate in PYM and Ti (57.41 and $56.44 \mathrm{~g} \mathrm{~kg}^{-1}$, respectively). Sinalbin is the precursor of p-HBITC, a compound characterized for its antimicrobial activity (Delaquis and Mazza 1995; Dufour et al. 2015; Ekanayake et al. 2012). Although many studies have investigated the antimicrobial effects of mustards for food preservation and biofumigation purposes, there is limited knowledge of the antimicrobial effects of p-HBITC as a potential biological fungicide against F. graminearum. For example, Ekanayake et al. (2006) demonstrated that IsoGard, a product based on p-HBITC extracted from moistened ground white mustard seed, had antimicrobial activity against pathogenic and spoilage bacteria, including Escherichia coli, Staphylococcus aureus, Campylobacter jejuni, Pseudomonas aeruginosa, Salmonella enteritidis, Listeria monocytogenes, Shigella boydii, and Clostridium perfringens. Furthermore, Quiles et al. (2015) investigated the potential of mustard-based botanicals to reduce aflatoxin contamination of wheat tortillas by Aspergillus parasiticus and observed that the gaseous allyl ITC from oriental mustard was more effective than p-HBITC from white mustard; however, substantial reduction in aflatoxin occurred with either treatment. Calmes et al. (2015) studied the mechanisms by which ITCs could cause fungal cell death using Alternaria brassicicola as a model organism. The authors demonstrated that exposure to these compounds led to reduced oxygen consumption rate, intracellular accumulation of reactive oxygen species, and mitochondrialmembrane depolarization. Smolinska et al. (2003) studied the effects of different ITCs as inhibitors of F. oxysporum. Propenyl-, ethyl-, benzyl-, and, in most cases, phenethyl-ITCs fully inhibited conidia and chlamydospore germination. However, the effects on mycelium growth were predominantly fungistatic and not fungitoxic, indicating that the mycelium may represent a more resistant stage of this fungus. In another study, the mycelium growth of $F$. graminearum was suppressed after the application of four alkenyl aliphatic ITCs (methyl-ITC, propenyl-ITC, butenyl-ITC, and pentenyl-ITC) or two aromatic ITCs (benzyl-ITC and 2phenylethyl-ITC) (Sarwar et al. 1998). In our study, the botanicals PYM and Ti, which contain sinalbin, completely suppressed the mycelium growth of all three strains of $F$. graminearum at a concentration of $2 \%$.

More alkaline environments reduce the stability of p-HBITC, resulting in the formation of a quinone that hydrolyses to ionic thiocyanate, $\mathrm{SCN}^{-}$(Borek and Morra 2005). To overcome this challenge in farming practices where mustard-based suspensions in water might be used, the product must be formulated to ensure the stability of p-HBITC. In addition to mustard-based botanicals, cereal farmers employing minimum tillage and, thus, encountering higher FHB disease pressure, could include mustard cover crops (e.g. Brassica juncea or Sinapis alba) in their rotation. After mulching the mustard crops, glucosinolates will be hydrolyzed and converted into bioactive ITCs. The latter would suppress various fungal structures in the life cycle of $F$. graminearum present in the field, including mycelium, perithecia, and conidia. In maizewheat rotations, an additional approach could be to cultivate mustard species as intercrops between maize rows. Thus, mustard residues after maize harvest might suppress the mycelium of $F$. graminearum growing on the maize residues and thus decrease the $F$. graminearum inoculum potential for the subsequent wheat crop.

The CGTs could be responsible for the antifungal activity observed against $F$. graminearum. CGTs can interact with membrane proteins of microorganisms by hydrogen bonding through their hydroxyl groups, which can result in changes in membrane permeability causing cell destruction (Burt 2004). Huang et al. (2012) studied the chemical composition of CG extract and the comparison between the efficacy of its main components and the whole extract in the inhibition of enamel demineralization. The authors concluded that gallic acid was the main demineralization-inhibiting component of $\mathrm{CG}$ and the effect was similar to that of the whole $\mathrm{CG}$ aqueous extract.
Tian et al. (2009b) evaluated the antibacterial activity of CGTs against Salmonella typhimurium and Bacillus cereus using a paper disc-based diffusion method. Hexa-hepta-galloylglucopyranoses (6-7-GGs), which were also identified in the CG powder used, showed remarkable antioxidant and antibacterial activities, suggesting that they could be utilized as food preservatives. In vitro studies on $F$. graminearum showed that solutions with $1 \%$ tannic acid or suspensions with 1\% CG inhibited conidia germination by 98 to $100 \%$ and mycelium growth by 75 to $80 \%$ (Forrer et al. 2014), which is in agreement with our findings. Moreover, Vogelgsang et al. (2013) conducted in vitro and field studies on the effect of aqueous extracts of botanical powders, including chamomile, meadowsweet, thyme, and CG against Microdochium majus. They found that CG had the most suppressive activity and completely inhibited mycelium growth and reduced conidia germination up to $97 \%$. However, for control of $\mathrm{FHB}$, the incomplete suppression of ascospore germination by $\mathrm{CG}$ suggests that it might be less effective in direct control strategies targeting flowering cereal heads.

Filamentous fungi are able to grow in a wide range of $\mathrm{pH}$ due to their efficient homeostatic mechanisms (Flaherty et al. 2003). Beyer et al. (2004) found similar conidia germination rates of $F$. graminearum at a $\mathrm{pH}$ ranging from 3 to 7 . Moreover, disease severity, DON content in grain, yield, and thousand-kernel weight of wheat plants were similar when treated with water $(\mathrm{pH}=7.8)$ or acidified water $(\mathrm{pH}=4.0)$ subsequent to artificial inoculation with conidia of $F$. graminearum and F. crookwellense (Forrer et al. 2014). Hence, we can surmise that the suppressive effects of the botanicals against $F$. graminearum in our study are not due to the slight decrease in $\mathrm{pH}$ by incorporating the botanicals in $\mathrm{dH}_{2} \mathrm{O}$ or in PDA.

In conclusion, we demonstrated that botanicals based on white mustard seed flour and CG were able to suppress or fully inhibit growth and development of $F$. graminearum using an array of in vitro bioassays. However, it should be noted that results of in vitro studies may not be directly transferable to the field. First, the interaction between the pathogen and plant is not taken into account. Second, under field conditions, crops are exposed to a wide range of pathogens and a mixture of several strains simultaneously, which is not the case in in vitro bioassays. Therefore, the antifungal effects of the botanicals must be tested in planta under both controlled environments and field conditions. Experiments should focus on direct control measures through applications of botanicals to flowering wheat heads. In addition, prevention strategies should be explored in reduced- or zero-tillage systems through botanical applications to crop residues in order to suppress inoculum of $F$. graminearum for the following crop.

\section{ACKNOWLEDGMENTS}

We thank A. Marty for technical assistance with the mycelium growth bioassay and G.S. Dunn Dry Mustard Millers, Canada, for providing Pure Yellow Mustard flour.

\section{LITERATURE CITED}

Amarasinghe, C. C., Tamburic-Ilincic, L., Gilbert, J., Brûlé-Babel, A. L., and Fernando, W. G. D. 2013. Evaluation of different fungicides for control of Fusarium head blight in wheat inoculated with 3ADON and 15ADON chemotypes of Fusarium graminearum in Canada. Can. J. Plant Pathol. 35: 200-208.

Antonissen, G., Martel, A., Pasmans, F., Ducatelle, R., Verbrugghe, E., Vandenbroucke, V., Li, S., Haesebrouck, F., Van Immerseel, F., and Croubels, S. 2014. The impact of Fusarium mycotoxins on human and animal host susceptibility to infectious diseases. Toxins 6:430-452.

Beyer, M., Roding, S., Ludewig, A., and Verreet, J. A. 2004. Germination and survival of Fusarium graminearum macroconidia as affected by environmental factors. J. Phytopathol. 152:92-97.

Blandino, M., Haidukowski, M., Pascale, M., Plizzari, L., Scudellari, D., and Reyneri, A. 2012. Integrated strategies for the control of Fusarium head blight and deoxynivalenol contamination in winter wheat. Field Crops Res. 133:139-149. 
Borek, V., and Morra, M. J. 2005. Ionic thiocyanate $\left(\mathrm{SCN}^{-}\right)$production from 4-hydroxybenzyl glucosinolate contained in Sinapis alba seed meal. J. Agric. Food Chem. 53:8650-8654.

Burt, S. 2004. Essential oils: Their antibacterial properties and potential applications in foods-A review. Int. J. Food Microbiol. 94:223-253.

Calmes, B., N'Guyen, G., Dumur, J., Brisach, C. A., Campion, C., Iacomi, B., Pigné, S., Dias, E., Macherel, D., Guillemette, T., and Simoneau, P. 2015. Glucosinolate-derived isothiocyanates impact mitochondrial function in fungal cells and elicit an oxidative stress response necessary for growth recovery. Front. Plant Sci. 6:414.

Dänicke, S., and Brezina, U. 2013. Kinetics and metabolism of the Fusarium toxin deoxynivalenol in farm animals: Consequences for diagnosis of exposure and intoxication and carry over. Food Chem. Toxicol. 60:58-75.

Dayan, F. E., Cantrell, C. L., and Duke, S. O. 2009. Natural products in crop protection. Bioorg. Med. Chem. 17:4022-4034.

Delaquis, P., and Mazza, G. 1995. Antimicrobial properties of isothiocyanates in food preservation. Food Technol. 49:73-84.

Djakpo, O., and Yao, W. 2010. Rhus chinensis and Galla chinensis-Folklore to modern evidence. Phytother. Res. 24:1739-1747.

D’Mello, J. P. F., Placinta, C. M., and Macdonald, A. M. C. 1999. Fusarium mycotoxins: A review of global implications for animal health, welfare and productivity. Anim. Feed Sci. Technol. 80:183-205.

Dufour, V., Stahl, M., and Baysse, C. 2015. The antibacterial properties of isothiocyanates. Microbiology 161:229-243.

Dweba, C. C., Figlan, S., Shimelis, H. A., Motaung, T. E., Sydenham, S., Mwadzingeni, L., and Tsilo, T. J. 2017. Fusarium head blight of wheat: Pathogenesis and control strategies. Crop Prot. 91:114-122.

Ekanayake, A., Kester, J. J., Li, J. J., Zehentbauer, G. N., Bunke, P. R., and Zent, J. B. 2006. IsoGard ${ }^{\mathrm{TM}}$ : A natural anti-microbial agent derived from white mustard seed. Acta Hortic. 709:101-108.

Ekanayake, A., Zoutendam, P. H., Strife, R. J., Fu, X., and Jayatilake, G. S. 2012. Development of white mustard (Sinapis alba L.) essential oil, a food preservative. Food Chem. 133:767-774.

Escrivá, L., Font, G., and Manyes, L. 2015. In vivo toxicity studies of Fusarium mycotoxins in the last decade: A review. Food Chem. Toxicol. 78: $185-206$

Flaherty, J. E., Pirttila, A. M., Bluhm, B. H., and Woloshuk, C. P. 2003. PAC1, a pH-regulatory gene from Fusarium verticillioides. Appl. Environ. Microbiol. 69:5222-5227.

Forrer, H. R., Hecker, A., Musa, T., Schwab, F., Bucheli, T. D., Wettstein, F. E., and Vogelgsang, S. 2014. Fusarium head blight control and prevention of mycotoxin contamination in wheat with botanicals and tannic acid. Toxins 6:830-849.

Gilbert, J., and Haber, S. 2013. Overview of some recent research developments in Fusarium head blight of wheat. Can. J. Plant Pathol. 35:149-174.

Huang, X. L., Liu, M. D., Li, J. Y., Zhou, X. D., and ten Cate, J. M. 2012. Chemical composition of Galla chinensis extract and the effect of its main component(s) on the prevention of enamel demineralization in vitro. Int. J. Oral Sci. 4:146-151.

Hyldgaard, M., Mygind, T., and Meyer, R. L. 2012. Essential oils in food preservation: Mode of action, synergies, and interactions with food matrix components. Front. Microbiol. 3:12.

Klittich, C. J. R., and Leslie, J. F. 1988. Nitrate reduction mutants of Fusarium moniliforme (Gibberella fujikuroi). Genetics 118:417-423.

Leplat, J., Friberg, H., Abid, M., and Steinberg, C. 2013. Survival of Fusarium graminearum, the causal agent of Fusarium head blight. A review. Agron. Sustain. Dev. 33:97-111.

Manstretta, V., Morcia, C., Terzi, V., and Rossi, V. 2016. Germination of Fusarium graminearum ascospores and wheat infection are affected by dry periods and by temperature and humidity during dry periods. Phytopathology 106:262-269.

Marin, S., Ramos, A. J., Cano-Sancho, G., and Sanchis, V. 2013. Mycotoxins: Occurrence, toxicology, and exposure assessment. Food Chem. Toxicol. 60: 218-237.

McMullen, M., Bergstrom, G., De Wolf, E., Dill-Macky, R., Hershman, D., Shaner, G., and Van Sanford, D. 2012. A unified effort to fight an enemy of wheat and barley: Fusarium head blight. Plant Dis. 96:1712-1728.

Osborne, L. E., and Stein, J. M. 2007. Epidemiology of Fusarium head blight on small-grain cereals. Int. J. Food Microbiol. 119:103-108.

Palazzini, J. M., Ramirez, M. L., Torres, A. M., and Chulze, S. N. 2007. Potential biocontrol agents for Fusarium head blight and deoxynivalenol production in wheat. Crop Prot. 26:1702-1710.
Palazzini, J. M., Yerkovich, N., Alberione, E., Chiotta, M., and Chulze, S. N. 2017. An integrated dual strategy to control Fusarium graminearum sensu stricto by the biocontrol agent Streptomyces sp. RC 87B under field conditions. Plant Gene 9:13-18.

Parry, D. W., Jenkinson, P., and Mcleod, L. 1995. Fusarium ear blight (scab) in small-grain cereals-A review. Plant Pathol. 44:207-238.

Pasquali, M., Beyer, M., Bohn, T., and Hoffmann, L. 2011. Comparative analysis of genetic chemotyping methods for Fusarium: Tri13 polymorphism does not discriminate between 3- and 15-acetylated deoxynivalenol chemotypes in Fusarium graminearum. J. Phytopathol. 159:700-704.

Prestera, T., Fahey, J. W., Holtzclaw, W. D., Abeygunawardana, C., Kachinski, J. L., and Talalay, P. 1996. Comprehensive chromatographic and spectroscopic methods for the separation and identification of intact glucosinolates. Anal. Biochem. 239:168-179.

Quarta, A., Mita, G., Haidukowski, M., Logrieco, A., Mule, G., and Visconti, A. 2006. Multiplex PCR assay for the identification of nivalenol, 3- and 15acetyl-deoxynivalenol chemotypes in Fusarium. FEMS Microbiol. Lett. 259:7-13.

Quiles, J. M., Manyes, L., Luciano, F. B., Manes, J., and Meca, G. 2015. Effect of the oriental and yellow mustard flours as natural preservative against aflatoxins B1, B2, G1 and G2 production in wheat tortillas. J. Food Sci. Technol. 52:8315-8321.

Quiles, J. M., Torrijos, R., Luciano, F. B., Manes, J., and Meca, G. 2018. Aflatoxins and A. flavus reduction in loaf bread through the use of natural ingredients. Molecules 23:E1638.

Romeo, L., Iori, R., Rollin, P., Bramanti, P., and Mazzon, E. 2018. Isothiocyanates: An overview of their antimicrobial activity against human infections. Molecules 23:E624.

Sarwar, M., Kirkegaard, J. A., Wong, P. T. W., and Desmarchelier, J. M. 1998. Biofumigation potential of brassicas. Plant Soil 201:103-112.

Schöneberg, A., Musa, T., Voegele, R. T., and Vogelgsang, S. 2015. The potential of antagonistic fungi for control of Fusarium graminearum and Fusarium crookwellense varies depending on the experimental approach. J. Appl. Microbiol. 118:1165-1179.

Sellam, A., Iacomi-Vasilescu, B., Hudhomme, P., and Simoneau, P. 2007. In vitro antifungal activity of brassinin, camalexin and two isothiocyanates against the crucifer pathogens Alternaria brassicicola and Alternaria brassicae. Plant Pathol. 56:296-301.

Shah, L., Ali, A., Yahya, M., Zhu, Y., Wang, S., Si, H., Rahman, H., and Ma, C. 2018. Integrated control of Fusarium head blight and deoxynivalenol mycotoxin in wheat. Plant Pathol. 67:532-548.

Smolinska, U., Morra, M. J., Knudsen, G. R., and James, R. L. 2003. Isothiocyanates produced by Brassicaceae species as inhibitors of Fusarium oxysporum. Plant Dis. 87:407-412.

Tian, F., Li, B., Ji, B. P., Yang, J. H., Zhang, G. Z., Chen, Y., and Luo, Y. C. 2009a. Antioxidant and antimicrobial activities of consecutive extracts from Galla chinensis: The polarity affects the bioactivities. Food Chem. 113: 173-179.

Tian, F., Li, B., Ji, B. P., Zhang, G. Z., and Luo, Y. C. 2009b. Identification and structure-activity relationship of gallotannins separated from Galla chinensis. LWT-Food. Sci. Technol. 42:1289-1295.

Trail, F. 2007. Fungal cannons: Explosive spore discharge in the Ascomycota. FEMS Microbiol. Lett. 276:12-18.

Trail, F. 2009. For blighted waves of grain: Fusarium graminearum in the postgenomics era. Plant Physiol. 149:103-110.

Trail, F., Xu, H. X., Loranger, R., and Gadoury, D. 2002. Physiological and environmental aspects of ascospore discharge in Gibberella zeae (anamorph Fusarium graminearum). Mycologia 94:181-189.

Vig, A. P., Rampal, G., Thind, T. S., and Arora, S. 2009. Bio-protective effects of glucosinolates-A review. LWT-Food. Sci. Technol. 42:1561-1572.

Vogelgsang, S., Bänziger, I., Krebs, H., Legro, R. J., Sanchez-Sava, V., and Forrer, H. R. 2013. Control of Microdochium majus in winter wheat with botanicals-From laboratory to the field. Plant Pathol. 62:1020-1029.

Yuen, G. Y., and Schoneweis, S. D. 2007. Strategies for managing Fusarium head blight and deoxynivalenol accumulation in wheat. Int. J. Food Microbiol. 119:126-130.

Zhang, Y., and Talalay, P. 1994. Anticarcinogenic activities of organic isothiocyanates: Chemistry and mechanisms. Cancer Res. 54:1976s-1981s.

Zhao, Y., Selvaraj, J. N., Xing, F., Zhou, L., Wang, Y., Song, H., Tan, X., Sun, L., Sangare, L., Folly, Y. M. E., and Liu, Y. 2014. Antagonistic action of Bacillus subtilis strain SG6 on Fusarium graminearum. PLoS One 9: e92486. 